\title{
Pain Management and Factors Associated with Its Severity among Post Surgical Patients Admitted in the Intensive Care Unit at Muhimbili National Hospital, Tanzania
}

\author{
Rebecca Samwel $^{1 *}$, Respicious Boniface ${ }^{1,2}$ \\ ${ }^{1}$ Department of Anaesthesiology, Muhimbili University of Health and Allied Sciences, Dar es Salaam, Tanzania \\ ${ }^{2}$ Department of Anaesthesiology, Muhimbili Orthopedic Institute, Dar es Salaam, Tanzania \\ Email: ^rebbyshijjy@gmail.com
}

How to cite this paper: Samwel, R. and Boniface, R. (2019) Pain Management and Factors Associated with Its Severity among Post Surgical Patients Admitted in the Intensive Care Unit at Muhimbili National Hospital, Tanzania. Open Journal of Anesthesiology, 9, 23-33.

https://doi.org/10.4236/ojanes.2019.92003

Received: December 7, 2018

Accepted: February 11, 2019

Published: February 14, 2019

Copyright $\odot 2019$ by author(s) and Scientific Research Publishing Inc. This work is licensed under the Creative Commons Attribution International License (CC BY 4.0).

http://creativecommons.org/licenses/by/4.0/

cC) (7) Open Access

\begin{abstract}
Background: Pain is the common experience among post operative patients admitted to the intensive care unit. Inadequate management can lead to undesired complications which can increase risk for morbidity and mortality. Objective: The aim of this study was to assess pain management and factors associated with its severity among post surgical patients admitted in intensive care unit at $\mathrm{MNH}$. Method: A prospective study was conducted from October 2017 to February 2018 involving a total of 123 post operative patients aged 18 years and above admitted to the surgical and obstetric intensive care units. Structured questionnaires were used to obtain the required perioperative information. Severity of pain was assessed by using the Numerical Rating Scale (NRS). Data was analyzed using SPSS version 23.0. Frequency, percentages, tables and charts were used to summarize the study findings. Bivariate analysis and multivariate logistic regression were done. P-value of $<0.05$ was considered significant. Results: The prevalence of severe post operative pain within 24 and 72 hours was $32.1 \%$ and $41.5 \%$ respectively. Pre operative use of analgesia (OR: $2.66, \mathrm{CI}: 1.15-6.12, \mathrm{P}$ value $=0.02$ ), abdominal surgery (OR: 4.12 CI: $1.12-15.88, \mathrm{P}$ value $=0.03$ ) and thoracic surgeries (OR; 7.42, CI: $1.54-35.88$, P value $=0.01)$ was significantly associated with severe pain. Age, sex, ASA class, duration of surgery, and level of education did not show significant association with pain severity. Opioids prescribed postoperatively were pethidine (70.7\%), morphine (11.4\%) and fentanyl (11.4\%). Other analgesics used were paracetamol (60.2\%) and diclofenac (22\%). Conclusion: The magnitude of post operative pain was high. Pre operative uses of analgesia, abdominal and thoracic surgery were the factors associated with severe pain.
\end{abstract}


Pethidine and paracetamol were the most prescribed drugs, however the prescription pattern used was inadequate to control pain.

\section{Keywords}

Post Operative Pain, Intensive Care Unit (ICU), Pain Severity, Prescription Pattern, Analgesia

\section{Background}

Post operative pain is a challenge encountered by most patients undergoing different surgical procedures. One review in the United Kingdom reported the overall incidence of severe post operative pain to be $11 \%$ [1]. For studies done in Africa, more than $50 \%$ of patients reported moderate to severe pain 24 hours post operatively [2] [3] [4] [5].

Several factors have shown to be associated with pain severity including age, education level and gender [6] [7] [8] [9]. Other factors include presence of higher pre operative pain, ASA physical status, pre operative use of analgesia, type of surgery especially thoracic surgery [7] [10], abdominal and orthopedic surgery [7] [10] [11], duration of surgery [6] [12] and type of anaesthesia [12].

Multimodal is the preferred modality of treatment and opioids are recommended for severe pain in intensive care units [13]. Non opioids like Non Steroidal Anti Inflammatory Drugs (NSAIDs) and Acetaminophen and other techniques like regional analgesia can all be used. Some medications can be used as adjuvant drugs; including ketamine, clonidine and dexmedetomidine. Non pharmacological methods like relaxation therapy and Transcutaneous Electrical Nerve Stimulation can also be used, however their effectiveness is not widely established [14].

Severe pain if not well managed can interfere with the patients' ambulatory status and make the patient at high risk for developing deep vein thrombosis, myocardial ischaemia, muscle wasting and respiratory complications such as lung infections and atelectasis [15] [16]. This study was designed to assess post operative pain management and the factors associated with its severity as a way forward in improving critical care services.

\section{Material and Methods}

\subsection{Study Design}

This was a prospective study conducted from October 2017 to February 2018.

\section{Study objectives}

The main objective was to assess pain management and factors associated with its severity among post operative patients admitted in ICU at MNH. The specific objectives were to determine the prevalence of severe post-operative pain, factors associated with pain severity and the prescription pattern used for post-operative pain. 


\section{Participant selection}

Convenience sampling method was used to obtain participants. Post operative patients aged 18 years and above were involved. Patients known to have severe cognitive disorders, ASA V and VI, those with severe traumatic brain injury defined by Glasgow coma scale $\leq 8$ and those who were non communicative beyond 72 hours were excluded.

\section{Study setting}

The study was conducted at Muhimbili National Hospital (MNH) surgical and obstetric intensive care units. Muhimbili is the national referral and teaching hospital in Tanzania, one of the countries in East Africa. It is the largest hospital in the country with a 1500 bed capacity, attending 1000 to 1200 outpatients per day and admitting 1000 to 1200 inpatients per week.

The surgical intensive care unit of this hospital had 14 beds and the obstetric intensive care unit had 4 beds. The surgical patients admitted in surgical ICU included those from general surgery, maxillofacial, otorhinolaryngology, obstetrics/gynecology, pediatric, urology and plastic surgery.

\subsection{Ethical Issues}

Ethical clearance was obtained from the Muhimbili University ethical research committee and the permission to conduct the study was also obtained from the Executive Director of the Muhimbili National Hospital. Consent was obtained for all patients who met the criteria for inclusion. Patients were free to withdraw anytime from the study without any charge.

This was not an interventional study. There were no invasive medical procedures performed to any patient. For patients who were found to have severe pain during data collection, the responsible health care worker was informed to provide the required analgesia. There was no payment given to patients who participated in this study and also patients were free to withdraw themselves anytime from the study without any charge against them.

\subsection{Tools}

Data was collected by using structured questionnaires and checklist. Pain was assessed using Numerical Rating Scale (NRS) which consists of numbers from 0 to 10 , with a mark at 0 for "no pain" and at 10 for "worst pain imaginable". For analysis purposes, in this study, 0 to 5 was considered "non severe pain" while the score from 6 to 10 was considered "severe pain".

\section{Data collection}

Data was collected by the principal investigator with two trained research assistants. Assessment was done within 72 hours post ICU admission when the patient was off sedation and able to communicate. Patients were helped by research assistants to fill the questionnaires, and those who were not able to put the mark on the scale, were allowed to say or point to the number correlating to the severity of pain they were feeling that time. 


\subsection{Data Analysis}

After data cleaning, analysis was done by using Statistical Package for Social Sciences (SPSS) version 23.0 program. Findings were presented in terms of frequency, percentages, tables and charts. Bivariate analysis and multivariate logistic regression were done. $\mathrm{P}$ value of $<0.05$ was considered significant.

\section{Results}

\subsection{Demographic Characteristics of the Study Participants}

A total of 128 participants were enrolled but only 123 were able to be assessed and analyzed $(\mathrm{N}=123)$. Of those who could be assessed 3 were unresponsive for more than 72 hours and 2 died before assessment.

From Table 1 below, $69.1 \%$ of patients aged below 50 years and $51.2 \%$ were male. $67.5 \%$ of them had primary education.

\subsection{Perioperative Characteristics of Participants}

During assessment, $39.8 \%$ of patients were on mechanical ventilators, $13 \%$ were on T-piece, $27.6 \%$ were on facemask and $19.5 \%$ were on room air. Among the assessed patients, 42 (34.1\%) had a history of analgesia use before surgery, ASA I and II patients were $84(68.3 \%)$ and $51(41.5 \%)$ had at least one history of surgery in the past. Intraoperatively, general anaesthesia was the main technique used (99.2\%). The median duration of surgeries was 170 minutes (IQR: 110 260). $95.7 \%$ of patients received analgesia intra operatively.

\subsection{Prevalence of Severe Pain}

In Figure 1, the prevalence of severe pain was found to be $32.1 \%$ and $41.5 \%$ within 24 and 72 hours respectively.

Within 24 hours; $27(32.1 \%)$ had severe pain while within 72 hours; 51 $(41.5 \%)$ reported severe pain.

Table 1. Demographic characteristics of study participants $(\mathrm{N}=123)$.

\begin{tabular}{ccc}
\hline Variable & Frequency n (\%) & Other comments \\
\hline Age (years) & $85(69.1)$ & Median = 41 (IQR: $30-55)$ \\
$18-50$ & $38(30.9)$ & \\
$>51$ & \\
Sex & $63(51.2)$ \\
Male & $60(49.8)$ \\
Female & \\
Education level & $2(1.6)$ \\
No formal & $83(67.5)$ \\
Primary school & $38(30.9)$ \\
Secondary school and above &
\end{tabular}

Key: IQR = Interquartile range. 


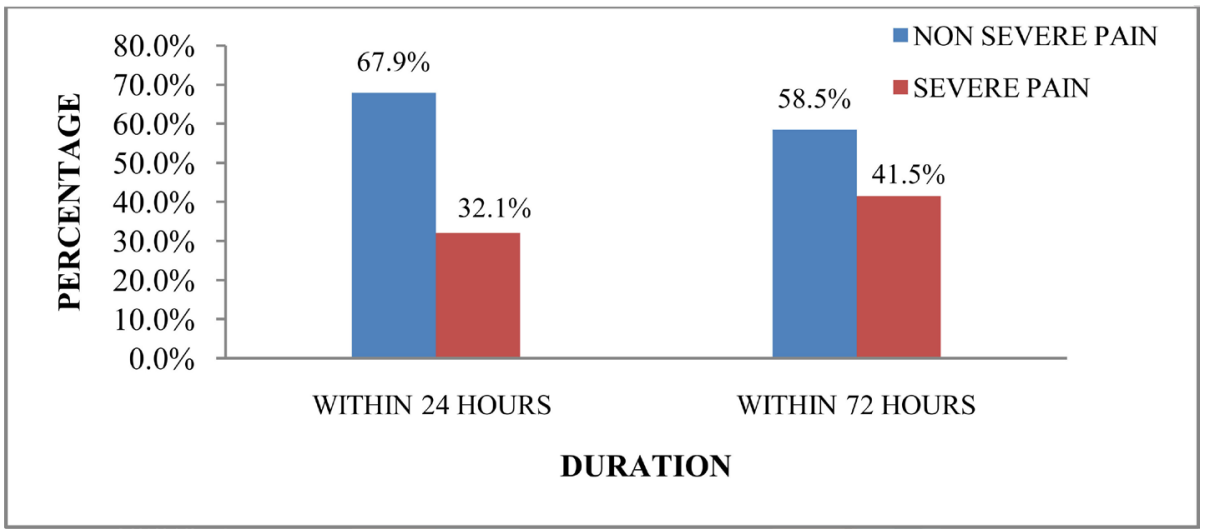

Figure 1. Bar chart showing the proportion of pain severity within twenty four hours $(\mathrm{N}=$ $84)$ and seventy two hours $(\mathrm{N}=123)$.

\section{Sources of pain}

In $98(79.7 \%)$ cases the main source of pain was reported to be incision wound. Others sources were endotracheal tubes $(8.1 \%)$, cannulas (1.6\%), nasogastric tubes $(0.8 \%)$ and others $(9.8 \%)$.

\subsection{Factors Associated with Pain Severity}

Factors which were assessed in our study were age, gender, level of education, ASA classification, pre operative use of analgesia, type and duration of surgery as summarized in the Table 2 below.

Factors which showed an association with pain severity on bivariate analysis were pre operative use of analgesia, type of surgery and duration of surgery (P-value < 0.05).

Multivariate logistic regression was then done to take care of confounders (pre operative use of analgesics, type of surgery and duration of surgery) and the findings were as summarized below Table 3.

Pre operative use of analgesia, abdominal and thoracic surgeries were significantly associated with severe pain. Patients who had history of pre operative use of analgesia were twice more likely to experience severe pain than those who didn't (OR: 2.66 CI: $1.15-6.34$, P value $=0.02$ ). Patients who underwent abdominal surgeries were four times more likely to experience severe pain than those who underwent maxillofacial surgeries (OR: 4.21, CI: $1.12-15.88$, P value $=0.03$ ) and those who underwent thoracic surgeries were 7 times more likely to experience severe pain than those who underwent maxillofacial surgery (OR: 7.42, CI: $1.54-35.88$, $\mathrm{P}$ value $=0.01)$. Duration of surgery and obstetric/gynecological surgeries didn't show significant association with pain severity (OR: 2.19, CI: 0.80 $4.11, \mathrm{P}$ value $=0.15)$ and $(\mathrm{OR}: 1.67, \mathrm{CI}: 0.34-8.24, \mathrm{P}$ value $=0.53)$ respectively.

\subsubsection{Types of Drugs Used}

Post operatively $72.4 \%(\mathrm{~N}=123)$ patients were kept on a multimodal regime of analgesics. The most used regimen was that of opioids with either diclofenac or paracetamol. Opioids were prescribed most, 115 (94.5\%) as compared to other 
Table 2. Summary of factors associated with pain severity on bivariate analysis $(\mathrm{N}=123)$.

\begin{tabular}{|c|c|c|c|c|}
\hline \multirow[b]{2}{*}{ Variable } & \multicolumn{2}{|c|}{ Pain severity } & \multirow[b]{2}{*}{ Total } & \multirow[b]{2}{*}{ P-value } \\
\hline & $\begin{array}{c}\text { Non severe } \\
\text { n (\%) }\end{array}$ & $\begin{array}{l}\text { Severe } \\
\text { n (\%) }\end{array}$ & & \\
\hline \multicolumn{5}{|l|}{ Age(years) } \\
\hline $18-50$ & $46(54.1)$ & $39(45.9)$ & 85 & \\
\hline$>51$ & $26(68.4)$ & $12(31.6)$ & 38 & 0.14 \\
\hline \multicolumn{5}{|l|}{ Sex } \\
\hline Male & $32(50.8)$ & $31(49.2)$ & 63 & \\
\hline Female & $40(66.7)$ & $20(33.3)$ & 60 & 0.07 \\
\hline \multicolumn{5}{|l|}{ Education level } \\
\hline No formal + primary & $51(60.0)$ & $34(40.0)$ & 85 & \\
\hline Secondary+ & $21(55.3)$ & $17(44.7)$ & 38 & 0.62 \\
\hline \multicolumn{5}{|l|}{ ASA class } \\
\hline$\leq \mathrm{II}$ & $49(49.2)$ & $35(50.8)$ & 84 & \\
\hline$\geq \mathrm{III}$ & $23(59.0)$ & $16(41.0)$ & 39 & 0.94 \\
\hline \multicolumn{5}{|l|}{ Pre op Analgesia } \\
\hline Yes & $19(45.2)$ & $23(54.8)$ & 42 & \\
\hline No & $53(65.4)$ & $28(34.6)$ & 81 & 0.03 \\
\hline \multicolumn{5}{|l|}{ Type of surgery } \\
\hline Abdominal & $24(49.0)$ & $25(51.0)$ & 49 & \\
\hline Thoracic & $6(35.3)$ & $11(64.7)$ & 17 & \\
\hline Obs/gyn & $11(68.8)$ & $5(31.2)$ & 16 & \\
\hline Other & $19(76.0)$ & $6(24.0)$ & 25 & \\
\hline Maxillofacial/dental & $12(75.0)$ & $4(25.0)$ & 16 & 0.02 \\
\hline \multicolumn{5}{|l|}{ Duration of surgery(min) } \\
\hline$\leq 180$ & $45(67.2)$ & $22(32.8)$ & 67 & \\
\hline$>180$ & $27(48.2)$ & $29(51.8)$ & 56 & 0.03 \\
\hline
\end{tabular}

Table 3. Summary of multivariate logistic regression analysis for pain severity.

\begin{tabular}{|c|c|c|c|c|c|}
\hline Variable & n (\%) & OR (CI) & AOR & $95 \% \mathrm{CI}$ & P-value \\
\hline \multicolumn{6}{|l|}{ Preop analgesia } \\
\hline No & $28(34.6)$ & 1 & 1 & & \\
\hline Yes & $23(54.8)$ & $2.29(1.07-4.90)$ & 2.66 & $1.15-6.12$ & 0.02 \\
\hline \multicolumn{6}{|l|}{ Type of surgery } \\
\hline Maxillofacial/dental & $4(25.0)$ & 1 & 1 & & \\
\hline Abdominal & $25(51.0)$ & $3.13(0.88-11.05)$ & 4.21 & $1.12-15.88$ & 0.03 \\
\hline Thoracic & $11(64.7)$ & $5.50(1.219-24.81)$ & 7.42 & $1.54-35.88$ & 0.01 \\
\hline Obs/gyn & $5(31.2)$ & $1.36(0.29-6.42)$ & 1.67 & $0.34-8.24$ & 0.53 \\
\hline Other & $6(24.0)$ & $0.95(0.22-4.07)$ & 1.44 & $0.30-6.34$ & 0.68 \\
\hline \multicolumn{6}{|l|}{ Duration of surgery } \\
\hline$\leq 180 \mathrm{mins}$ & $22(32.8)$ & 1 & 1 & & \\
\hline$\geq 181 \mathrm{mins}$ & $29(51.8)$ & $2.19(1.06-4.57)$ & 1.81 & $0.80-4.11$ & 0.15 \\
\hline
\end{tabular}

Key: $\mathrm{OR}=$ Odds ratio, $\mathrm{AOR}=$ Adjusted Odds Ratio, $\mathrm{CI}=$ Confidence interval, mins $=$ minutes. 
drugs. Pethidine was prescribed more frequently (70.9\%) than morphine (11.4\%) and fentanyl (11.4\%). Paracetamol was the second most prescribed drug (60.2\%), followed by diclofenac (22.0\%).

The comparison between multimodal and single therapy use with pain severity was done and the findings were summarized in the chart below.

From these findings it appears that $42.7 \%$ of patients who were kept on multimodal therapy had severe pain as compared to those who used single therapy $(38.2 \%)$. However this difference was not significant $(\mathrm{P}$-value $=0.6)($ Figure 2$)$.

\subsubsection{Route of Administration}

Intravenous and intramuscular were the main routes of administration used in the intensive care unit. For morphine, the route used most was intravenous (78.6\%) and for pethidine the intramuscular route was used most (86.2\%). Fentanyl and paracetamol were given intravenously in all cases while diclofenac was given intramuscularly in all cases.

\subsubsection{Dose of the Prescribed Drugs}

Morphine was prescribed at the dose of 10 milligrams in most cases $(71.4 \%)$, other doses used were 2 milligrams and 5 milligrams. The prescribed dose for pethidine was 50 milligrams (14.9\%) and 100 milligrams (85.1\%). Fentanyl was prescribed at 50 micrograms (14.3\%) and 100 micrograms (85.7\%). For diclofenac 75 milligrams was the only strength used and for paracetamol the doses used were 500 milligrams (2.7\%) and 1000 milligrams (97.3\%).

\section{The prescribed drug frequency}

Morphine was prescribed in PRN ("pro re nata"-when necessary) regimen in $7.1 \%, 6$ hourly (28.6\%) and 8 hourly (64.3\%). Pethidine was prescribed more frequently at an interval of 6 hours (58.6\%) and 8 hours (39\%). For fentanyl, the frequencies used were 4 hourly (28.6\%), 6 hourly (42.8\%) and 8 hourly (28.6\%). The frequencies used for paracetamol were 6 hourly $(33.4 \%)$ and 8 hourly $(66.2 \%)$ and for diclofenac the 8 hourly frequency was used in all the prescriptions.

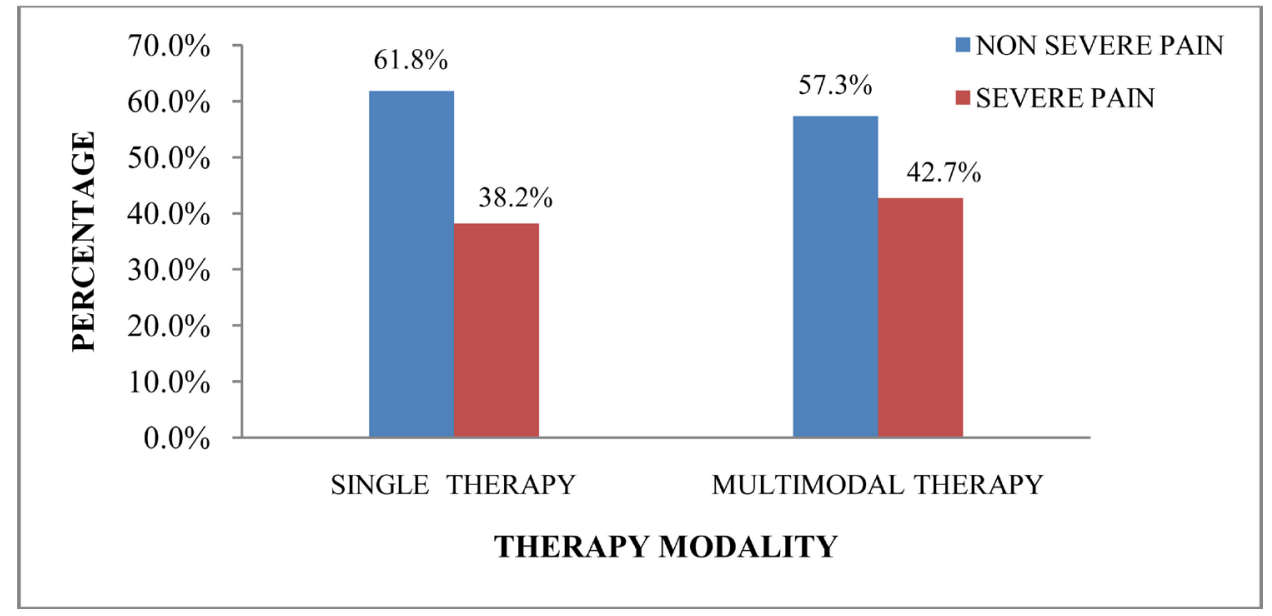

Figure 2. Bar chart showing the adequacy of pain control for patients on multimodal therapy and those on single therapy (P-value $=0.6)$. 


\section{Discussion}

Post operative pain is challenging especially in critically ill patients. In our study, the proportions of patients who reported severe pain within 24 to 72 hours post operative were significantly high, $30 \%$ to $40 \%$ respectively. These high proportions could be due to the prescriptions pattern which appeared to be similar in majority of patients regardless of the variations existing among them including different sizes and depth of the incision wounds as the reported main source of the pain. Two studies done in Tanzania revealed similar findings, one study revealed a proportion of $40.3 \%$ for moderate to severe pain [5] and in another study more than half of patients reported moderate to worst pain after 24 hours [4]. Other studies also found similar findings [2] [3].

By comparing the 24 hours assessment and those of within 72 hours it appeared that majority of patients reported severe pain even after 24 hours. This could be due to the fact that most of the ICU procedures like wound dressing, drains and tube removal were observed to be done after 24 hours. Although it was also observed that little analgesia was sometimes given during these procedures, there was no clear established protocol for procedural pain management, and most of these procedures were done by nurses who cannot prescribe. Another observation was that most of the prescriptions were written to cover the first 24 hours only and a variable amount of time elapsed before the next prescriptions which were mostly composed of single therapy only.

Different factors can influence pain severity. In our study pre operative use of analgesia was significantly associated with severe pain. Patients who use of analgesia for prolong duration might have chronic pain which causes them to have increase in sensitivity to pain (hyperalgesia). These patients also have an increase in analgesia requirement therefore relatively higher dosages are needed to control their pain severity. Similar findings were obtained in the studies done by Auburun et al. and Kinjo et al. [9] [12].

Abdominal and thoracic surgeries showed to be associated with severe pain as well. The reason for this association could be related to the intensity, site and duration of the procedure. Most of these procedures take long time as a result tissues are significantly exposed to injury which causes severe pain. These sites are also prone to procedures like coughing and breathing which exaggerate pain as well. It is usual for these procedures to be accompanied with tubes for drainage which also adds pain. The studies by Chia Y. et al. and Hui Yun et al. showed a significant association between thoracic surgeries and post operative pain severity as well [7] [10].

Duration of surgery didn't show an association with pain severity in our study although the confidence limits were very wide so certainly for some patients it was associated with increased pain.

In our study, multimodal analgesia with at least two drugs combination was used in up to $70 \%$ of patients. This was quite interesting and positive step toward proper pain management as far as the advantages of multimodal therapy were 
concerned; however the significant difference on pain severity between patients who were treated with multimodal and those on single therapy could not be demonstrated. This could probably be due to the prescription pattern which did not individualize severe pain enough and the choice of drugs which were used as part of the multimodal therapy in combination with opioids. Drugs used most were diclofenac and paracetamol which are usually effective in controlling mild to moderate pain.

Intravenous route is the preferred route of drug administration for ICU patients. In our study, the route used most to administer pethidine was intramuscular. This route is associated with erratic absorption hence difficult to predict the drug effect and therefore inadequate pain control can result. Inadequate pain control was the main finding in other studies where intramuscular route was a major route of administration [2] [3] [4] [5].

Intermittent dosages were used for almost all patients; the dose amount was estimated according to the estimated body weight. Although the strength used for pethidine which were fixed to $50 \mathrm{mg}$ and $100 \mathrm{mg}$ were reasonable for a normal adult person, variations among patients were supposed to be taken into account. Due to the fact that the duration of action for pethidine ranges between 2 to 4 hours and pethidine was mostly administered 6 to 8 hourly, patients were at higher risk of experiencing pain in between doses. Intermittent administration of analgesics in patients who have undergone major surgery may not be adequate and therefore continuous infusion or patient controlled analgesia can provide better control of pain. Studies in which intermittent administration were done inadequate pain control was reported [3] [4] [5] while in those where Patient Controlled administration systems were used, better control of pain was reported [17].

\section{Study limitations}

This was a single center study therefore generalization was difficult; however these findings can be useful in improving post operative pain management in other intensive care units.

\section{Conclusion}

Pain is a challenge existing in most post operative patients admitted in intensive care unit and the magnitude of post operative pain is high. Severity of pain can be increased with the pre-operative use of analgesia, abdominal and thoracic surgeries. Pethidine and paracetamol were the most prescribed drugs post-operatively. Pethidine was prescribed intramuscularly in high proportion at the strength of $50 \mathrm{mg}$ and $100 \mathrm{mg}$, and the interval of 6 to 8 hours. Paracetamol was prescribed intravenously at the interval of 8 hours.

\section{Availability of Data and Material}

The dataset used during this study is available from the corresponding author on request. 


\section{Acknowledgements}

The author would like to acknowledge Dr Respicious Boniface, Research assistants and participants for making this study successful.

\section{Conflicts of Interest}

The authors declare no conflict of interest.

\section{Funding}

This was a non funded study; the principle investigators used their own funds to support the whole project.

\section{Authors Contributions}

Conceptualization: RS.

Supervision: RB.

Methodology: RS, RB.

\section{References}

[1] Dolin, S.J., Cashman, J.N. and Bland, J.M. (2002) Effectiveness of Acute Postoperative Pain Management: I. Evidence from Published Data. British Journal of Anaes thesia, 89, 409-423. https://doi.org/10.1093/bja/89.3.409

[2] Fawole, A.A. and Kolawole, I. (2003) Post Operative Pain Management Following Caesarean Section in University of Ilorin Teaching Hospital. West African Journal of Medicine, 22, 305-309.

[3] Ocitti, E.F. and Adwok, J.A. (2000) Post-Operative Management of Pain Following Major Abdominal and Thoracic Operations. East African Medical Journal, 77, 299-302.

[4] Makia, M.I. (2004) Post Operative Pain Therapy Prescribing Patterns and Adequacy of Post Operative Analgesia, at Muhimbili National Hospital Dar es Salaam, Tanzania. University of Dar es Salaam, Dar es Salaam.

[5] Herbert, G., Masigati, I. and Chilonga, K.S. (2014) Postoperative Pain Management Outcomes among Adults Treated at a Tertiary Hospital in Moshi, Tanzania. Tanzania Journal of Health Research, 16, 1-9.

[6] Bradshaw, P., Hariharan, S. and Chen, D. (2016) Does Preoperative Psychological Status of Patients Affect Postoperative Pain? A Prospective Study from the Caribbean. British Journal of Pain, 10, 108-115. https://doi.org/10.1177/2049463716635680

[7] Hui Yun, V., Abrishami, A., Peng, P. and Wong, J.C.F. (2009) Predictors of Postoperative Pain and Analgesic Consumption. Anesthesiology, 111, 657-677. https://doi.org/10.1097/ALN.0b013e3181aae87a

[8] Singh, J.A. and Sherine Gabriel, D.L. (2008) The Impact of Gender, Age, and Preoperative Pain Severity on Pain After TKA. Clinical Orthopaedics and Related Research, 466, 2717-2723. https://doi.org/10.1007/s11999-008-0399-9

[9] Kinjo, S., Sands, L.P., Lim, E., Sudeshna, P. and Leung, J.M. (2012) Prediction of Post Operative Pain Using Path Analysis in Older Patients. Journal of Anesthesia, 26, 1-8. https://doi.org/10.1007/s00540-011-1249-6

[10] Chia, Y., Chow, L., Hung, C., Liu, K., Ger, L. and Wang, P. (2002) Gender and Pain 
upon Movement Are Associated with the Requirements for Postoperative Patient-Controlled IV Analgesia: A Prospective Survey of 2298 Chinese Patients. Canadian Journal of Anesthesia, 49, 249-255. https://doi.org/10.1007/BF03020523

[11] Kalkman, C.J., Visser, K., Moen, J., Bonsel, G.J., Grobbee, D.E. and Moons, K.G.M. (2003) Preoperative Prediction of Severe Postoperative Pain. International Association for the Study of Pain, 105, 415-423. https://doi.org/10.1016/S0304-3959(03)00252-5

[12] Auburun, F., Valade, N., Coriat, P. and Riou, B. (2008) Predictive Factors of Severe Postoperative Pain in the Postanesthesia Care Unit. Anesthesia and Analgesia, 106, 1535-1541. https://doi.org/10.1213/ane.0b013e318168b2ce

[13] Barr, J., Fraser, G.L., Puntillo, K., Ely, E.W., Gélinas, C., Dasta, J.F., et al. (2013) Clinical Practice Guidelines for the Management of Pain, Agitation, and Delirium in Adult Patients in the Intensive Care Unit. Critical Care Medicine, 41, 263-306. https://doi.org/10.1097/CCM.0b013e3182783b72

[14] Carrillo-Torres, O., Ramirez-Torres, M.A. and Mendiola-Roa, M.A. (2016) Update on the Assessment and Treatment of Pain in Critically Ill Patients. Revista Médica del Hospital General de México, 79, 165-173. https://doi.org/10.1016/j.hgmx.2016.05.012

[15] Layzell, M. (2008) Current Interventions and Approaches to Postoperative Pain Management. British Journal of Nursing, 17, 414-420. https://doi.org/10.12968/bjon.2008.17.7.29059

[16] Butterworth, J.F., Mackey, D.C. and Wasnick, J.D. (2013) Clinical Anesthesiology. 5th Edition, McGraw Hill Education, New York, 1087-1103.

[17] Brodner, G., Mertes, N., Buerkle, H., Marcus, M.A.E. and Van Aken, H. (2000) Acute Pain Management: Analysis, Implications and Consequences after Prospective Experience with 6349 Surgical Patients. European Journal of Anaesthesiology, 17, 566-575. https://doi.org/10.1046/j.1365-2346.2000.00738.x 